\title{
Human-Based Human Milk Fortifier as Rescue Therapy in Very Low Birth Weight Infants Demonstrating Intolerance to Bovine-Based Human Milk Fortifier
}

\author{
Amanjot Sandhu, Sharla Fast, Kari Bonnar, Ronald John Baier, and Michael Narvey
}

\begin{abstract}
Objective: To describe the results of utilizing a human milk-based human milk fortifier (HMHMF) as rescue therapy to meet nutritional requirements in very low birth weight and preterm infants demonstrating feeding intolerance to bovine-based human milk fortifier (BHMF) in the Canadian Neonatal Intensive Care Unit (NICU) setting.

Materials and Methods: At two Level III NICUs in Winnipeg, MB, Canada, a rescue protocol was implemented to provide HMHMF for infants demonstrating intolerance to BHMF. To qualify for rescue, infants were required to experience two episodes of significant gastrointestinal (GI) symptoms associated with fortification with BHMF. A case series report was conducted retrospectively examining the success of rescue therapy, growth rates, protein, and calorie intakes before and after initiation of HMHMF in seven infants.

Results: Seven infants (birth weight $723 \pm 247 \mathrm{~g}$, gestation $25.3 \pm 3.4$ weeks) were treated with rescue fortification with HMHMF. All infants were transitioned off parenteral nutrition (PN) without relapse of GI symptoms. Growth rate, protein, and calorie intakes improved with the use of HMHMF.

Conclusions: Very low birth weight and preterm infants with GI intolerance to BHMF were successfully rescued with use of HMHMF. Improvements in growth were achieved without need for supplementation with PN through achievement of sufficient enteral calorie and protein intakes.
\end{abstract}

Keywords: fortifier, low birth weight, premature, feeding intolerance, human milk

\section{Introduction}

$\mathbf{H}$ UMAN MILK IS the ideal source of nutrition for infants. Human milk alone does not meet the complex nutritional requirements of very low birth weight and preterm infants. 1,2 When compared with formula fed to infants, human milk has been shown to reduce rates of necrotizing enterocolitis (NEC) in preterm infants. ${ }^{3}$

However, preterm infants require fortification of human milk to achieve optimal growth and developmental outcomes, which until recently has been exclusively produced from bovine sources. Fortification of human milk with a bovine-based human milk fortifier (BHMF) may lead to variable degrees of gastrointestinal (GI) symptoms that, owing to similarity with early stages of NEC, may result in a period of withholding enteral feeds or milk fortification. ${ }^{4}$ Withholding either milk fortification or enteral feeds yields suboptimal nutrition for the infant or extends the duration of parenteral supplementation.
Since 2012, a human milk-based human milk fortifier (HMHMF) (Prolacta ${ }^{\circledR}$; Prolacta Bioscience, Inc., City of Industry, CA) became available after it was approved by Health Canada. This product offers the opportunity to provide fortification that may result in an exclusive human milk diet for the majority of an infant's neonatal intensive care unit (NICU) stay. We introduced the use of HMHMF as a rescue treatment for those with failure to tolerate traditional BHMF. The objective of this study was to describe the efficacy of HMHMF as rescue therapy for infants in the NICU setting with demonstrated intolerance to BHMF. We describe the outcomes of these infants and offer an alternative approach of targeted rescue treatment for centers similar to ours that are experiencing resistance to recommended usage of HMHMF because of financial restrictions.

\section{Materials and Methods}

A retrospective case series was conducted regarding the clinical outcomes of rescue fortification using HMHMF.

Department of Neonatology, University of Manitoba, Winnipeg, Manitoba, Canada.

(C) Amanjot Sandhu et al., 2017; Published by Mary Ann Liebert, Inc. This is an Open Access article distributed under the terms of the Creative Commons Attribution License, which permits unrestricted use, distribution, and reproduction in any medium, provided the original work is properly cited. 
Patients were admitted to one of two Level III NICUs in Winnipeg, Manitoba, Canada. Ethics approval was granted by the Health Ethics Research Board of the University of Manitoba.

All patients in this cohort received expressed breast milk $(\mathrm{EBM})$ or donor breast milk (DBM) during their time under study. In each case, the only source of cow's milk protein was BHMF. In our NICUs, we use a Mead Johnson BHMF as standard of care. Although evidence exists supporting the use of liquid BHMF rather than powdered forms, our NICUs only have access to powdered forms, as this is the only form approved by Health Canada.

All infants were treated with a minimum of 72 hours of minimal enteral feedings and then advanced as tolerated by $30 \mathrm{~mL} /(\mathrm{kg} \cdot$ day $)$ until full feedings were reached of 150 $165 \mathrm{~mL} /(\mathrm{kg} \cdot$ day $)$. BHMF was added once infants reached a minimum of $80 \mathrm{~mL} /(\mathrm{kg} \cdot$ day $)$ of enteral feeding.

The rescue protocol involved identifying patients in the NICU setting that demonstrated intolerance to BHMF. Intolerance was defined by GI signs and symptoms (significant abdominal distension, increased residuals, emesis, and hematochezia) occurring after addition of BHMF and was considered a diagnosis of exclusion. In each case, withdrawal of BHMF led to improvement in symptoms and upon rechallenge with BHMF similar symptoms recurred, necessitating cessation again. After two failures, a written request from the dietitian for HMHMF was directed to the Division Head of Neonatology (M.N.) for permission to provide the product.

Information on clinical characteristics was abstracted from the medical record. Detailed information on feeding was collected, including the day of life (DOL) when trophic feeds were started, when feeds were incremented, when BHMF was introduced (first and second trials), occurrences of GI symptoms, and HMHMF initiation and weaning. Average enteral calorie and protein intakes before and after the introduction of HMHMF were also documented. Growth rates were calculated for the period from initiation of BHMF to initiation of $\mathrm{HMHMF}, 7$ days before initiation of HMHMF and from initiation of HMHMF to the day weaning of HMHMF began. Growth rates, calorie, and protein intakes before and during HMHMF were compared by using paired $t$-tests. Data are presented as mean \pm standard deviation or median and interquartile range where appropriate.

\section{Results}

\section{Case report}

An $835 \mathrm{~g}$ male infant was born at 25 weeks to a 36-year-old primigravida after an otherwise unremarkable pregnancy. He was ventilated after birth because of apnea and respiratory distress syndrome. Apgar scores were 1, 2, and 2 at 1, 5, and 10 minutes. Trophic feedings $(10 \mathrm{~mL} /(\mathrm{kg} \cdot$ day $)$ of DBM were started within a few hours of admission and continued until DOL 5 when feedings were advanced using an increment of $20-30 \mathrm{~mL} /$ (kg-day). Fortification with BHMF (one package per $50 \mathrm{~mL}$ EBM or DBM) was started on DOL 10 at an enteral feeding volume of $\sim 90 \mathrm{~mL} /(\mathrm{kg} \cdot$ day $)$ and increased on DOL 12 to one package per $25 \mathrm{~mL}$ EBM/DBM. Within 24 hours of reaching this level of fortification, he developed anuria, abdominal distention, and an increase in feeding residuals that led to him being placed nothing by mouth (NPO) on DOL 13. Feedings with EBM were restarted after 24 hours of withholding enteral feeds.

A second trial of BHMF occurred on DOL 16 with a further increase to one package to $25 \mathrm{~mL}$ EBM on DOL 18. This introduction of BHMF coincided with treatment of a patent ductus arteriosus with indomethacin. GI symptoms recurred (grossly distended abdomen, faint bowel sounds, and loose green stool), and he was placed NPO briefly and fed solely with EBM for the next 3 days.

Since the second attempt to introduce BHMF was associated temporally with patent ductus arteriosus (PDA) treatment, a third attempt at adding BHMF was made, and again resulted in emesis on DOL 21. His feeds were then fortified with HMHMF on DOL 22 at +6 and increased to +8 on DOL 30 . He remained stable on HMHMF feeds for 14 days and was slowly weaned back to BHMF. Nutrition was improved dramatically with the introduction of HMHMF (enteral intake $120 \mathrm{kcal} /(\mathrm{kg} \cdot$ day) vs. $65 \mathrm{kcal} /(\mathrm{kg} \cdot$ day $) ; 3.8 \mathrm{~g} /(\mathrm{kg} \cdot$ day $)$ vs. $1.2 \mathrm{~g} /(\mathrm{kg} \cdot$ day $)$ protein $)$. He gained $30-34 \mathrm{~g} /(\mathrm{kg} \cdot$ day $)$ while on HMHMF compared with $15 \mathrm{~g} /(\mathrm{kg} \cdot$ day $)$ before fortification.

Table 1. Primary Outcomes Both Before and After Initiation of Human Milk-Based Human Milk Fortifier

\begin{tabular}{|c|c|c|c|c|c|c|c|}
\hline \multirow[b]{2}{*}{ Case } & \multirow[b]{2}{*}{$\begin{array}{l}\text { Gestational age } \\
\text { and birth weight }\end{array}$} & \multirow[b]{2}{*}{$\begin{array}{l}\text { Duration of } \\
\text { minimum } \\
\text { enteral } \\
\text { feedings } \\
\text { (days) }\end{array}$} & \multicolumn{3}{|c|}{ Before initiation of $H M H M F^{\mathrm{a}}$} & \multicolumn{2}{|c|}{ After $H M H M F^{\mathrm{b}}$} \\
\hline & & & $\begin{array}{c}\text { Overall } \\
\text { growth } \\
\text { rate before } \\
\text { HMHMF } \\
(\mathrm{g} /(\mathrm{kg} \cdot \text { day }))\end{array}$ & $\begin{array}{c}\text { Growth rate } \\
7 \text { days before } \\
\text { HMHMF } \\
(g /(k g \cdot d a y))\end{array}$ & $\begin{array}{c}\text { Mean kcal } \\
(\mathrm{kcal} /(\mathrm{g} \cdot \text { day })) / \\
\text { mean protein } \\
\text { intake } \\
(\mathrm{g} /(\mathrm{kg} \cdot \text { day }))\end{array}$ & $\begin{array}{l}\text { Growth rate } \\
\text { on } \mathrm{HMHMF} \\
(\mathrm{g} /(\mathrm{kg} \cdot \mathrm{day}))\end{array}$ & $\begin{array}{c}\text { Mean kcal } \\
(\mathrm{kcal} /(\mathrm{g} \cdot \text { day })) / \\
\text { mean protein } \\
\text { intake } \\
(\mathrm{g} /(\mathrm{kg} \cdot \text { day }))\end{array}$ \\
\hline 1 & 24 weeks $710 \mathrm{~g}$ & 5 & 8 & 11 & $109 / 3.75$ & 16 & $123 / 3.5$ \\
\hline 2 & 24 weeks $460 \mathrm{~g}$ & 5 & 10 & 16 & $105 / 3.5$ & 17 & $123 / 3.7$ \\
\hline 3 & 24 weeks $590 \mathrm{~g}$ & 5 & 13 & 15 & $130 / 3.6$ & 17 & $140 / 4.4$ \\
\hline 4 & 23 weeks $510 \mathrm{~g}$ & 5 & 7 & $29^{c}$ & $118 / 3$ & 12 & $137 / 4.3$ \\
\hline 5 & 24 weeks $770 \mathrm{~g}$ & 4 & -12 & 1.9 & $129 / 4.4$ & 19 & $113 / 3.5$ \\
\hline 6 & 33 weeks $1190 \mathrm{~g}$ & 3 & 3.4 & 3.9 & $79 / 1.3$ & 19 & $162 / 4.5$ \\
\hline 7 & 25 weeks $835 \mathrm{~g}$ & 5 & 30 & -7 & $70 / 2.0$ & 32 & $126 / 4.1$ \\
\hline
\end{tabular}

${ }^{\text {a }}$ The period of time from initiation of minimal enteral feedings, through both episodes of intolerance to BHMF and ending at the date of initiation of HMHMF.

${ }^{\mathrm{b}}$ The period of time from the first day of HMHMF to the first day the infant was weaned from HMHMF.

${ }^{\mathrm{c}}$ Growth rate exaggerated, clinically attributed to edema.

BHMF, bovine based human milk fortifier; HMHMF, human milk-based human milk fortifier. 
Table 2. Total Nutrient (Enteral and Parenteral) INTAKE AND GROWTH BEFORE AND DURING ForTIFICATION WITH HUMAN MILK-BASED Human Milk Fortifier

\begin{tabular}{lccc}
\hline & $\begin{array}{c}\text { Before } \\
\text { HMHMF }^{\mathrm{a}}\end{array}$ & $\begin{array}{c}\text { During } \\
\text { HMHMF }^{\mathrm{b}}\end{array}$ & $\mathrm{p}$ \\
\hline $\begin{array}{c}\text { Caloric intake } \\
(\mathrm{kcal} /(\mathrm{kg} \cdot \mathrm{day}))\end{array}$ & $106 \pm 23$ & $131 \pm 17$ & 0.079 \\
$\begin{array}{c}\text { Protein intake } \\
(\mathrm{kcal} /(\mathrm{kg} \cdot \text { day }))\end{array}$ & $3.1 \pm 1.1$ & $4.1 \pm 0.4$ & 0.082 \\
$\begin{array}{c}\text { Growth rate } \\
(\mathrm{g} /(\mathrm{kg} \cdot \text { day }))\end{array}$ & $10.0 \pm 11.6$ & $16.8 \pm 1.1$ & 0.224 \\
\hline
\end{tabular}

${ }^{a}$ Before HMHMF refers to the period of time from initiation of minimal enteral feedings until the first day of HMHMF was administered.

${ }^{\mathrm{b}}$ During HMHMF refers to the period of time from initiation of HMHMF to the first day the infant began being weaned from HMHMF.

HMHMF, human milk-based human milk fortifier.

Seven infants who received HMHMF after a minimum of two episodes of intolerance to BHMF are reported (Table 1). Mean birth weight was $723 \pm 246 \mathrm{~g}$ and gestation was $25.3 \pm 3.5$ weeks. All received $72-120$ hours of trophic feeding before incrementing feeds. BHMF was initially introduced at a median of 10 (interquartile range [IQR]; 8-18) DOL. The first episode of intolerance to BHMF occurred on day 14 (IQR; 8 $18), 2.6 \pm 2.0$ days after the first introduction. BHMF was reintroduced at a median of 22 (IQR; 15-24) DOL. The second episode of intolerance to BHMF occurred on day 23 (IQR; 18 27), 2.6 \pm 2.7 days after subsequent introduction. Fortification with HMHMF was started at DOL 31 (IQR; 24-48).

All infants tolerated fortification with HMHMF with no infant requiring early discontinuation of fortification with HMHMF. There was a trend toward increased caloric and protein intake with HMHMF but no difference in growth with HMHMF supplementation (Table 2). Six of seven infants experienced an increase in growth rate after introduction of HMHMF compared with the week before the starting of this fortification. In the fortification period, this was achieved enterally compared with a mixture of enteral and parenteral intake previous to fortification. One patient was clinically edematous, which may have masked improved growth.

\section{Discussion}

HMHMF (Prolacta) is now available in Canada as a means of providing fortification to either EBM or DBM to achieve an exclusive human milk diet. The manufacturer recommends starting supplementation after the first few DOL continuing until 33 or 34 weeks postmenstrual age. Although the product has been demonstrated to be well tolerated and may decrease duration of parenteral nutrition $(\mathrm{PN})$ and rates of NEC, it does come at a greater up front financial cost than BHMF. When taking downstream health cost savings into consideration, these initial expenditures may be offset and, in turn, become overall health cost savings when factoring in the total cost of a patient's stay. ${ }^{5,6}$

Long-term health consequences such as retinopathy of prematurity and bronchopulmonary dysplasia have been associated to occur at a lower rate on an exclusively human milk-based diet. ${ }^{7}$ Owing to concerns over such unbudgeted expenditures in our hospital, we were unable to initiate use of the product as suggested and developed a local guideline for use as a rescue treatment for those infants who demonstrate probable intolerance to BHMF.

In our seven patients, rescue therapy resulted in weaning from PN and achievement of goal protein and caloric intakes. Furthermore, no negative GI symptoms reoccurred during supplementation with HMHMF. Although in our case report tolerance of feedings with HMHMF was noted with the infant, at some point in this period of fortification of HMHMF the PDA for this child decreased in size from moderate to small in size. We cannot exclude the possibility that the elimination of diastolic steal of intestinal blood flow allowed for tolerance of feeding on this occasion.

The use of HMHMF did show a positive growth trend, although it was not associated with statistically significant improvements in the growth rate, mean caloric intake, or mean protein intake compared with the period before implementation. This must be put in context, however, that each of these infants required interruption of feedings and PN before rescue. Given that we saw no differences in growth velocity and that protein and caloric intakes were similar before and after the introduction of HMHMF, we speculate that the addition of HMHMF allowed for increased provision of nutrients preserving the growth rate when transitioning off of PN. With this perspective, we see the rescue approach as beneficial in that infants were successfully transitioned from a parenterally supplemented approach to one that was completely enteral for nutrition.

Shortening the duration of intravenous access has the added benefit of reducing the risk of complications of infection and thrombosis from the use of intravascular access. PN is required to provide sufficient calories and protein intake for those infants who cannot be fed by the enteral route. This comes with an additional cost in failure to provide direct stimulation of the enteral tract. Frequent monitoring of blood parameters, increased risk of infection, and higher costs associated with PN can be avoided by using enteral feeding with HMHMF in infants demonstrating intolerance to BHMF.

The seven infants in our study all demonstrated evidence of intolerance to powdered BHMF as demonstrated by symptoms that appeared on challenge and rechallenge with bovine sources of nutrition that ultimately abated with a change to an exclusive human milk diet. Upon rechallenge with bovine sources of nutrition after weaning from HMHMF, some of these infants' bovine intolerance was improved. Thus, it is possible that a period of treatment with HMHMF allowed for healing of the intestine and or increase in surface area, resulting in tolerance of feedings.

Although it is possible that time could have contributed to the tolerance these infants had demonstrated, it may be important to state their intolerance before this time had lapsed and was bridged with enteral nutrition, which allowed the gut to continue being stimulated. Further research is needed to examine whether such growth and healing do occur after transition to an exclusive human milk diet once the GI tract is matured.

In these seven patients, it is clear that they had the ability to tolerate enteral feeding, but were part of a small number of infants in our units who had increased sensitivity to BHMF. We suggest that such infants, if identified, could be spared a number of the aforementioned adverse outcomes if recognized early and provided rescue HMHMF.

To our knowledge, this is the first description of using HMHMF as a rescue therapy feeding protocol for preterm 
infants. In so describing, we offer an alternative to units who are unable to justify the routine use of HMHMF for all infants because of restrictions imposed by the current lack of up front funding and competing budget dollars.

As this was a descriptive retrospective study, the data are subject to the interpretation of those reviewing the charts and the consistency of documentation in medical records. The time the infant remained on BHMF before demonstrating intolerance was variable in our seven cases. What remains consistent is that all seven infants demonstrated two episodes of intolerance within 0-5 days (range of days) on first challenge and 0-8 days upon subsequent rechallenge with $\mathrm{BHMF}$ that improved with removal of BHMF and substitution with HMHMF. Based on this initial report, we believe further research is warranted using HMHMF as rescue therapy in a larger prospective cohort of infants to determine effects on GI development and measures of growth.

\section{Conclusions}

Very low birth weight and premature infants, who demonstrated feeding intolerance to BHMF with GI symptoms, were successfully rescued with use of HMHMF and can continue enteral feeding and maturation of their GI tract without compromising their growth rate and protein or calorie intakes. Once these infants were rescued, HMHMF provided the first successful enteral source of nutrition, particularly goal calories and protein.

\section{Acknowledgments}

The authors would like to thank the parents of our NICU patients for trusting in our use of HMHMF, when all other fortifiers were not tolerated by their infants. Thank you to management and staff for their teamwork and hard work in collecting, preparing, procuring, and all other daunting aspects of this new product. Thank you to the Winnipeg NICU staff teams and Child Health leadership teams for supporting us in the use of HMHMF to help provide the best patient care we can. Lastly, thank you to Prolacta Bioscience for supporting our dietitians as we learned how to use this new product to nutritionally rescue patients.

\section{Disclosure Statement}

No competing financial interests exist.

\section{References}

1. Schanler RJ. Evaluation of the evidence to support current recommendations to meet the needs of premature infants: The role of human milk. Am J Clin Nutr 2007;85: 625-628.

2. Ziegler EE. Meeting the nutritional needs of the low-birthweight infant. Ann Nutr Metab 2011;58 Suppl 1:8-18.

3. Quigley M, Mcguire W. Formula versus donor breast milk for feeding preterm or low birth weight infants (Review). Cochrane Libr 2014;1-92. doi: 10.1002/14651858.CD002971 .pub3.www.cochranelibrary.com.

4. Sullivan S, Schanler RJ, Kim JH, et al. An exclusively human milk-based diet is associated with a lower rate of necrotizing enterocolitis than a diet of human milk and bovine milk-based products. J Pediatr 2010;156:562-567.

5. Ganapathy V, Hay JW, Kim JH. Costs of necrotizing enterocolitis and cost-effectiveness of exclusively human milkbased products in feeding extremely premature infants. Breastfeed Med 2012;7:29-37.

6. Assad M, Elliott MJ, Abraham JH. Decreased cost and improved feeding tolerance in VLBW infants fed an exclusive human milk diet. J Perinatol 2015;36:1-5.

7. Hair AB, Peluso AM, Hawthorne KM, et al. Beyond necrotizing enterocolitis prevention: Improving outcomes with an exclusive human milk-based diet. Breastfeed Med 2016; 11:70-74.

Address correspondence to: Amanjot Sandhu, MD University of Manitoba MS361J 820 Sherbrook Street Winnipeg R3A 1R9 Manitoba Canada

E-mail: umsandhu@myumanitoba.ca Michael Narvey, MD, FRCPC, FAAP

University of Manitoba MS361J 820 Sherbrook Street Winnipeg R3A 1R9 Manitoba Canada

E-mail: mnarvey@hsc.mb.ca 